\title{
Osteopathic manual therapy (OMT) in treatment of gastroesophageal reflux disease (GERD)
}

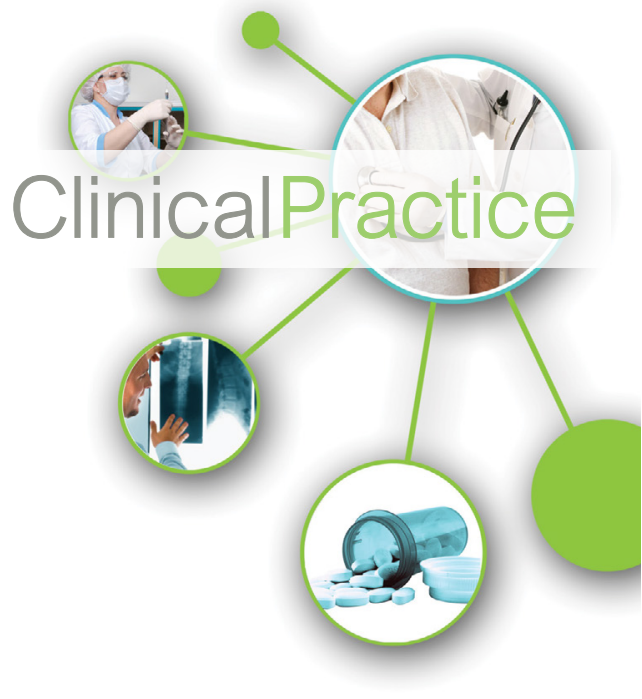

\begin{abstract}
Background: Osteopathic Manual Therapy (OMT) is a non-pharmaceutical and none-invasive treatment, designed to treat Gastroesophageal Reflux Disease (GERD). The aims were to determine a Minimum Efficacy Dose (MED) and the longterm effect of OMT in the treatment of GERD.
\end{abstract}

Materials and method: The study was performed as a two-dimensional, between-patient Response Surface Pathway (RSP) designed multicenter study with "Number of OMTs" and "Treatment Interval" as interventional variables. The main response variable was the percent reduction in the sum of the five GERD-scores from baseline. Three patients on the first design level received six OMT with five days' interval. The number of OMTs and treatment interval in the second $(n=5)$ and third design levels $(n=7)$ were based on the results of the previous design level. The mean age and duration of GERD were 50.2 and 10.9 years, ranging from 25.7 to 75.7 years and 0.2 to 36.3 years, respectively.

Results: The percent reduction in GERD-score increased with increasing number of OMTs and time intervals, but flattered out after four OMTs and three to four days treatment interval. The estimated MED of OsMT was three treatments with two days interval resulting in a GERD-score reduction of $62.2 \%$ (95\% Cl: 50.3-80.1\%). All the five GERD-symptoms and consequently the mean GERD-score were significantly reduced after the first two OMTs, one week, three and 12 months after the last treatment $(p<<0.01)$. One week after last OMT, the GERD-score reduced with $75 \%$ and $33 \%$ symptoms free patients. At one-year follow-up, the reduction was $63.3 \%$ with $46.7 \%$ had no GERD-symptoms.

Conclusion: Anti-reflux OMT significantly decreases the GERD-symptoms and the use of PPI. 46.7 percent of the patents had no GERD symptoms one year after treatment. The estimated MED of OMT was three treatments with two days (48 hours) interval.

\section{Keywords: Gastroesophageal reflux disease (GERD), osteopathic manual therapy (OMT), patient}

\section{Introduction}

Gastroesophageal Reflux Disease (GERD) has an obvious negative impact on Quality Of Life (QOL). The prevalence is increasing worldwide [1-3] and may even be reflected in the economy of the society $[4,5]$. Today, the most commonly used class of drugs for GERD is Proton Pump Inhibitors (PPI). This approach is not curative and frequently requires continuous medication. In addition, patients are advised to make lifestyle changes, which may reduce the symptoms of reflux. The only approved curative treatment today is surgical procedures such as fundoplication. Surgery is mainly used in patients with severe GERD-symptoms often combined with insufficient PPI effect. However, surgery is invasive very difficult to reverse and carries a risk for serious adverse effects.
A special designed anti-reflux OMTprocedure combining traction of the cardia, mobilization of the diaphragm and thoracic spine with posture correction was applied in a study of 22 endoscopically examined GERD patients [6]. A postural correction consisted of an extension of columna, carried out daily by the patient. The patient was supine positioned on a soft pillow for 15 minutes, which was located in the thoracic-lumbar region. A total osteopathic examination was conducted. Areas in the body that could affect the lesion region were manually treated. The cardia region may be influenced by viscero-somatic reflexes or mechanical tensions in the diaphragm area. Treatment techniques performed were adapted to each patient, as described in the osteopathic literature [7]. The reduction in the total number
Kjell E Bjørnæs ${ }^{1}$, Geir Elvbakken ${ }^{1}$, Bård Dalh $\varnothing i^{1}$, Tor Harald Garberg ${ }^{1}$, Joachim Kaufmann ${ }^{1}$, Espen Glomsrød ${ }^{1}$, Ola Reiertsen ${ }^{2}$ and Stig Larsen ${ }^{3 *}$

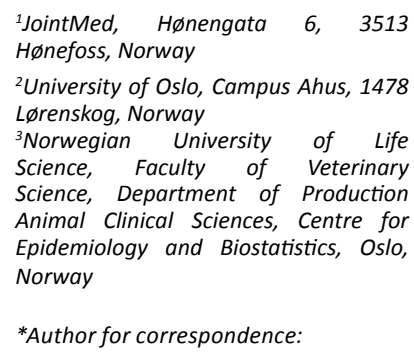

${ }^{2}$ University of Oslo, Campus Ahus, 1478 Lørenskog, Norway

${ }^{3}$ Norwegian University of Life Science, Faculty of Veterinary Science, Department of Production Animal Clinical Sciences, Centre for Epidemiology and Biostatistics, Oslo, Norway

*Author for correspondence:

stig.larsen@nmbu.no 
of GERD-symptoms was significant, and "moderate" or "good" effect was detected in $77.3 \%$ of the patients. However, the lack of controls reduced the strength of these results. Recently a randomized, double-blinded, and placebo-controlled study in 58 patients of OMT in the treatment of GERD was published [8]. All the GERD-symptoms were significantly reduced one week after the last treatment in the OMT group $(n=44)$ compared to no reduction in the control group $(n=14)$. Twelve percent of the OMT patients were symptom-free, and 75\% reported above a $50 \%$ reduction in symptoms. "Acid in the mouth" and "Heartburn" was reduced by $81 \%$. The OMT-procedure was well described $[6,8]$, but neither the OMT dose nor the long-term effect was clarified. The dose of OMT was defined as the number of OMTs applied and the interval between each treatment needed to obtain a qualified reduction in the symptoms.

The main aim of this study is to determine a Minimum Efficacy Dose (MED) of OMT by assessing the GERD-symptoms one week after the last treatment. Furthermore, the effects on GERD-symptoms, GERD-score, and use of PPI three and twelve months after treatment were assessed.

\section{Material and Methods}

The study population consisted of GERD patients, passed the age of 18 years. Patients with hiatus hernia $\geq 5 \mathrm{~cm}$ and patients suffering from gastric ulcer, cancer and uncontrolled bacterial, viral, fungal or parasite infection were excluded. The patients were recruited from and treated at five Norwegian osteopathic clinics with Halden in south, Bergen in west, Hønefoss in east, Trondheim in the mid and Alta in north. The study was approved by the regional ethical committee, and the patients gave informed consent to participate. Ten female and five male GERD patients were included [9]. The mean and range of age were 50.2 years $(25.7$ to 75.7$)$, duration of disease 10.9 years, $(0.2$ to 36.3 ) and body-mass-index $24.3 \mathrm{~kg} / \mathrm{m}^{2}$ (17.637.7). Thirteen patients responded on PPI with moderate to good effect. One patient had no effect of PPI and one had severe Adverse Events (AE) and did not use PPI at the time of inclusion in the study. Additionally, one patient had previously received acupuncture with moderate effect and one received alternative treatment with good effect. Two patients did not use any medication or received other GERD-treatment prior or during the study. Six patients reported muscular and skeleton disease whereof one received osteopathic treatment. Seven patients had no concomitant disease.

The study was conducted as a twodimensional, randomized between-patient RSP three-level designed multicentre trial $[9,10]$ with "Number of OMT's" and "Interval between the treatments" as intervention variables. The degree of "Heartburn", "Chest pain", "Acid in the mouth", "Epigastric pain" and "Thoracic pain" were recorded by the patients on a $10 \mathrm{~cm}$ Visual Analogue Scale (VAS) [11]. Registration of the five GERD-symptoms was performed by the patients at baseline, before every new treatment, one week, three and 12 months after the last treatment. The sum of the five GERDsymptoms and percent reduction from baseline to one week after the last OMT was the main response. Patients obtaining a reduction of at least $50 \%$ were classified as a responder to the treatment. As secondary variables "Sleeping problem", "Digestive problem" and "Vomiting" were recorded by the patients at all visit by using VAS. Additionally, the use of PPI and other reflux treatment were recorded.

Statistical analysis: Continuously distributed variables were expressed by mean values with 95\% Confidence Interval (CI) [12]. As an index of dispersion, Standard Deviations (SD) was given. Categorized variables were expressed in contingency tables [13]. The development in an intervention variable was performed by using polynomial regression analysis with the other intervention variable as a covariate [14]. The percent reduction in the sum of GERDsymptoms assumed ordinal in both the two interventional variable and the probability increases monotonically over the interventional levels. Isotonic regression was used for the estimation of MED [15-17].

\section{Results}

The sum of GERD-symptoms fell significantly $(\mathrm{p}<<0.01)$ from baseline until four OMTs and was stabilized (FIGURE 1A). Additionally, the sum of symptoms was significantly reduced with increasing duration between the OMTs until an interval of three to four days (FIGURE 1B). The MED of OMT in The treatment of GERD was estimated to three OMT's with an interval of two days or 48 hours, 
the estimated percent reduction of GERDsymptoms was $62.2 \%$ (95\% CI: 50.3-80.1\%).

The mean sum of GERD-symptoms was significantly reduced after the first two OMTs $(\mathrm{p}<<0.01)$, and one week, three months and one year after the last OMTs (FIGURE 2). One week after the last OMT, the sum of GERDsymptoms fell with $75 \%$. Thirteen of the 15 patient had a reduction above $50 \%$ and hence classified as responders. Five of the patients or
33\% had no GERD-symptoms (TABLE 1). The mean reduction in GERD-symptoms was nearly stable the following three months after the last OMT with 11 patients as responders and the fraction of patients with no GERDsymptoms increased to $46.7 \%$. One year after the last OMT, the mean reduction in GERDsymptoms was $63.3 \%$. Ten of the 15 patients were classified as responders to the treatment, and the number of symptom-free patients was unchanged from the three months follow-up.
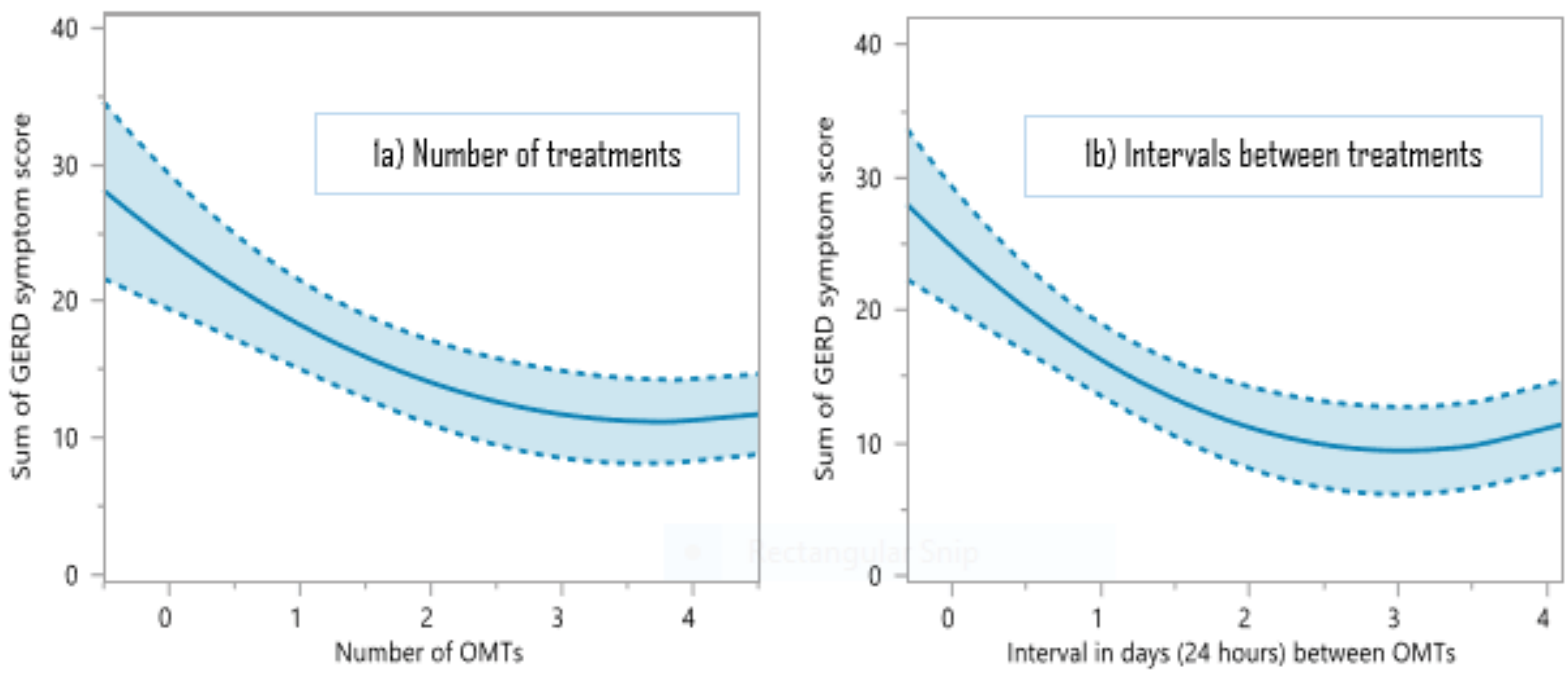

FIGURE 1. Sum of GERD-symptoms as a function (A) of treatment number corrected for treatment interval in days and

(B) of interval durations corrected for a number of treatment. The shaded area shows the $95 \%$ confidence boundary.

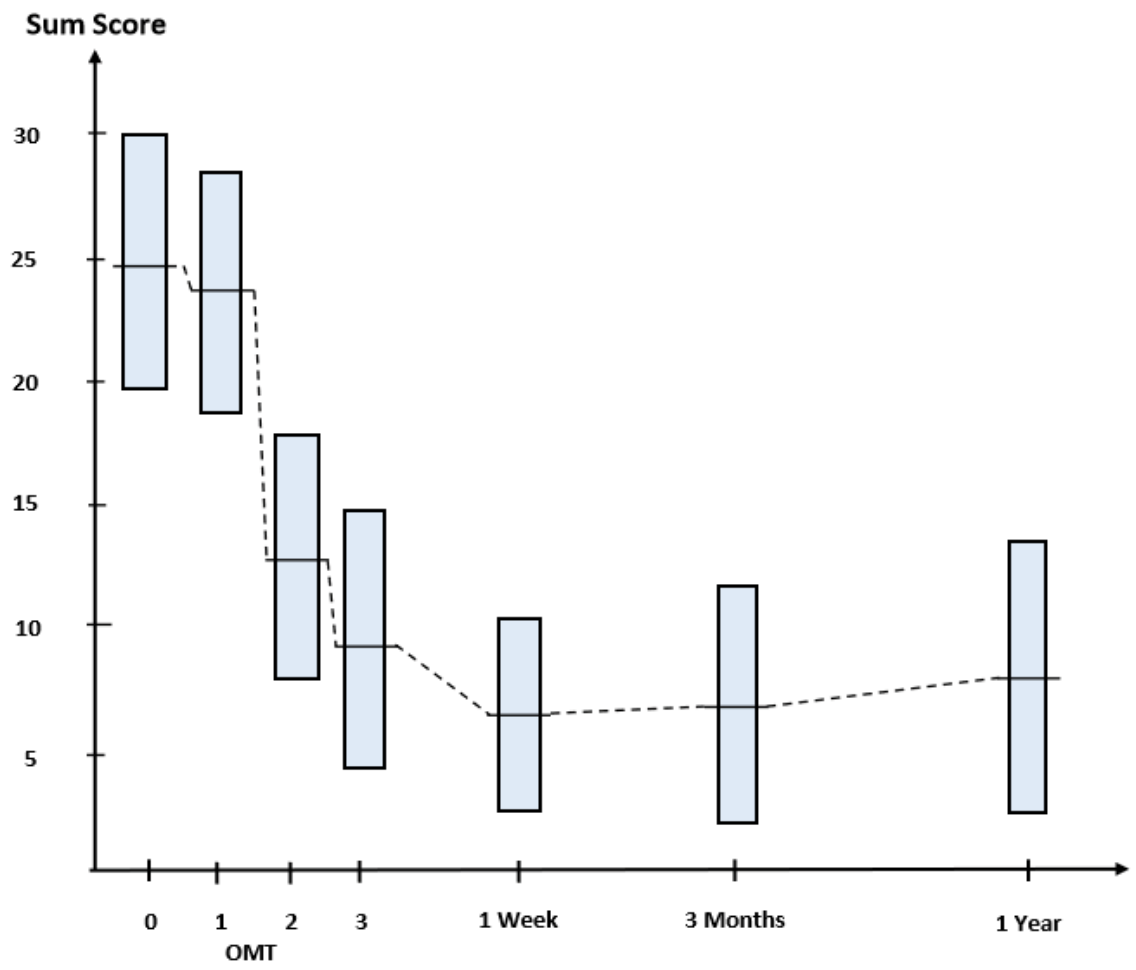

FIGURE 2. Development in degree of sum GERD-symptoms in number of OMTs during the treatment and one, week, three months and one year after last OMT. The results expressed by mean values with $95 \%$ Confidence Intervals. The blue columns indicate the confidence intervals and the horizontal line crossing the columns shows the mean values. 
The reduction in mean percent responder was due to two patients obtaining other diseases after three months follow-up (FIGURE 2), which indirectly resulted in the return of the GERDsymptoms. By leaving these two patients out from the analysis, the reduction was $77.4 \%$ and $53.8 \%$ was symptom-free after one year.

The five GERD-symptoms were all significantly reduced above $56 \%$ one week after the last OMT (TABLE 2). The largest reduction detected in "Thoracic pain" with $84.2 \%$ and the smallest in "Acid in the mouth" (TABLE 1). At three months follow-up, all the five GERD-symptoms were reduced with at least $60 \%$. The symptom with the largest reduction was "Epigastric pain" with $80.4 \%$ and the smallest obtained in "Heartburn" (TABLE 1). The situation was nearly unchanged one year after the last OMT. Despite the two patients obtaining other disease influencing GERD, all symptoms were significantly reduced from baseline $(\mathrm{p}<<0.01)$ with more than $50 \%$. The largest reduction was detected in "Heartburn" with $65.5 \%$ and smallest in "Thoracic pain".

Of other symptoms related to GERD, "Sleeping problems" was reported at baseline by nine patients, "Digestive problems" by eight and "Vomiting Problem" by five patients (TABLE 3). One week and three months after last OMT, two patients reported "Sleeping problems and "Vomiting Problem". After one year, four respectively two patients reported these two symptoms, respectively. All the five patients reporting "Digestive problems" at baseline were free of the symptom one week, three months and one year after the last OMT. The degree of "Sleeping problems" was reduced with $89.1 \%$, $84.9 \%$, and $56.0 \%$ one week, three months and one year after last OMT, respectively (TABLE 3). The degree of "Vomiting Problem" was reduced by $77.3 \%$ one week after last OMT and $80.9 \%$ and $92 \%$ at three months and one-year follow-up. The GERD-related symptoms were reported by 12 patients at baseline but only by two patients one week after the last OMT, three patients at three months follow-up and five

\begin{tabular}{|c|c|c|c|c|c|}
\hline $\begin{array}{l}\% \text { reduction from } \\
\text { baseline }\end{array}$ & $\begin{array}{l}\text { GERD Symptoms } \\
\text { (No. of patients) }\end{array}$ & $<50 \%$ & $>50-75 \%$ & $>75-95 \%$ & $\geq 95 \%$ \\
\hline \multirow{6}{*}{$\begin{array}{l}\text { One week after the last } \\
\text { OMT }\end{array}$} & Heartburn (12) & 4 & 3 & 2 & 3 \\
\hline & Chest Pain (14) & 4 & 1 & 3 & 6 \\
\hline & Acid in mouth (12) & 5 & 2 & 0 & 5 \\
\hline & Epigastric Pain (13) & 4 & 2 & 2 & 5 \\
\hline & Thoracic Pain (11) & 1 & 3 & 2 & 5 \\
\hline & Sum GERD symptoms & 2 & 4 & 4 & 5 \\
\hline \multirow{6}{*}{$\begin{array}{l}\text { Three months after the } \\
\text { last OMT }\end{array}$} & Heartburn (12) & 4 & 1 & 1 & 6 \\
\hline & Chest Pain (14) & 4 & 0 & 1 & 9 \\
\hline & Acid in mouth (12) & 4 & 2 & 0 & 6 \\
\hline & Epigastric Pain (13) & 1 & 4 & 2 & 6 \\
\hline & Thoracic Pain (11) & 4 & 1 & 0 & 6 \\
\hline & Sum GERD symptoms & $4^{1}$ & 3 & 1 & 7 \\
\hline \multirow{6}{*}{$\begin{array}{l}\text { One year after the last } \\
\text { OMT }\end{array}$} & Heartburn (12) & 3 & 0 & 1 & 8 \\
\hline & Chest Pain (14) & 5 & 1 & 1 & 7 \\
\hline & Acid in mouth (12) & 4 & 0 & 0 & 8 \\
\hline & Epigastric Pain (13) & 3 & 2 & 3 & 5 \\
\hline & Thoracic Pain (11) & 5 & 0 & 0 & 6 \\
\hline & Sum GERD symptoms & $5^{2}$ & 1 & 2 & 7 \\
\hline
\end{tabular}

TABLE 2. Reduction in the five GERD symptoms from baseline to one week, three months and one year after last OMT. The results expressed by mean values and $95 \%$ confidence intervals in bracket.

\begin{tabular}{|l|c|c|c|c|}
\hline \multirow{2}{*}{$\begin{array}{l}\text { GERD Symptom } \\
\text { assessed on a 10cm VAS }\end{array}$} & \multirow{2}{*}{ No. of pat. } & \multicolumn{3}{|c|}{ Reduction in degree of symptoms after last OMT } \\
\cline { 3 - 5 } & & One week & Three months & One year \\
\hline Heartburn & 12 & $3.7(1.6-5.8)$ & $4.0(1.7-6.3)$ & $4.5(1.9-7.1)$ \\
\hline Chest Pain & 14 & $4.4(2.7-6.1)$ & $4.8(2.8-6.9)$ & $3.9(1.7-6.1)$ \\
\hline Acid in mouth & 12 & $2.6(1.3-4.7)$ & $3.3(1.6-5.0)$ & $3.3(1.1-5.5)$ \\
\hline Epigastric Pain & 13 & $4.8(2.8-6.8)$ & $5.2(4.0-6.5)$ & $4.5(2.1-6.9)$ \\
\hline Thoracic Pain & 11 & $5.5(4.1-6.6)$ & $4.4(2.0-6.8)$ & $3.8(0.7-6.8)$ \\
\hline
\end{tabular}


TABLE 3. Reduction in degree of "Sleeping problem", "Digestive problem" and "Vomiting" one week, three months and one year after the last OMT. The results expressed by mean values with $95 \%$ confidence intervals.

\begin{tabular}{|l|c|c|c|c|}
\hline \multirow{2}{*}{$\begin{array}{l}\text { GERD related Symptom assessed on } \\
\text { a 10cm VAS }\end{array}$} & \multirow{2}{*}{ No of patients } & \multicolumn{3}{|c|}{ Reduction in degree of related symptoms after last OMT } \\
\cline { 3 - 5 } & & One week & Three months & One year \\
\hline Sleeping problems & 9 & $4.6(2.3-6.8)$ & $4.1(2.0-6.2)$ & $2.8(0.0-5.6)$ \\
\hline Digestive problems & 5 & $3.8(0.0-7.8)$ & $3.8(0.0-7.8)$ & $3.8(0.0-7.8)$ \\
\hline Vomiting problems & 8 & $4.3(1.9-6.6)$ & $3.4(1.8-5.0)$ & $4.4(2.4-6.3)$ \\
\hline Sum related symptoms & 12 & $7.4(4.2-10.7)$ & $6.9(3.8-10.1)$ & $5.7(1.3-10.0)$ \\
\hline
\end{tabular}

\section{TABLE 4. Reduction in use of anti-reflux treatment after last OMT.}

\begin{tabular}{|c|c|c|c|c|c|}
\hline \multirow{2}{*}{ Visit } & \multirow[b]{2}{*}{ Frequency } & \multicolumn{4}{|c|}{ Baseline } \\
\hline & & None & When needed & Daily used & Total \\
\hline \multirow{4}{*}{ One week after last OMT } & None & 2 & 4 & 4 & 10 \\
\hline & When needed & 0 & 2 & 1 & 3 \\
\hline & Daily used & 0 & 0 & 2 & 2 \\
\hline & Total & 2 & 6 & 7 & 15 \\
\hline \multirow{4}{*}{ Three months after last OMT } & None & 2 & 4 & 3 & 9 \\
\hline & When needed & 0 & 0 & 3 & 3 \\
\hline & Daily used & 0 & 2 & 1 & 3 \\
\hline & Total & 2 & 6 & 7 & 15 \\
\hline \multirow{4}{*}{ One year after last OMT } & None & 2 & 5 & 3 & 10 \\
\hline & When needed & 0 & 1 & 0 & 1 \\
\hline & Daily used & 0 & 0 & 4 & 4 \\
\hline & Total & 2 & 6 & 7 & 15 \\
\hline
\end{tabular}

patients after one year. (TABLE 3).

The use of PPIs reported by the patients was significantly $(\mathrm{p}<0.01)$ reduced from baseline to one week, three and 12 months after last OMT (TABLE 4). Two patient did not use PPI due to lack of efficacy and severe AE neither at baseline nor during the study and follow-up. One week after last OMT only two patients permanently used PPI and eight patients have stopped all anti-reflux treatment. Three patients reporting daily use of anti-reflux treatment at three months follow-up and further to four one year after the last OMT. At three months follow-up, seven patients had stopped anti-reflux treatment and eight after one year.

\section{Discussion}

The present study supports the results from both our previous publications $[6,8]$ and others [18]. The sum of GERD-symptoms reduces significantly already after two treatments and increasing with increased numbers of OMTs. However, the effect flattered out after three to four treatments indicating that most of the OMT effect then is obtained. In the papers describing the anti-reflux OMT procedure, the number of OMTs varied from two to 10 , but in the placebo-controlled study, the number of treatments was standardized to three $[6,8]$. This seems to be nearby the optimal number.
The prevalence of responders to OMT one week after last treatment in this study [8] was $75 \%$ compared to $28.6 \%$ in the placebo group. This is identical to the response rate obtained in the present study in which the number of treatments varied from two to six. In the two previous studies, the patients were followed only one week after the last OMT, compared to the present study in which the patients were followed up with new investigation first three months and finally 12 months after the last OMT. If the effect has disappeared only a short time after the end of treatment, this results would not have been more than interesting. However, the effect was nearly unchanged after three and 12 months. In the group of patients classified as a responder to OMT, the sum of GERD symptoms was reduced with 75\%, 70\%, and $63 \%$ one week, three and 12 months after the last treatment, respectively. However, the prevalence of symptom-free patients increases from $33 \%$ one week after the last OMT to nearly $50 \%$ one year later.

The duration between each OMT treatment is central in Osteopathy. It is stated that the body has to recover between each intervention [19]. In the previous GERD-studies [6,8] this factor has varied from one to two weeks. However, the present study indicated that this duration should be substantially smaller. The MED was estimated to three OMTs with a duration between each 
treatment of only two days. Additionally, the effected flattered out with an interval between three and four days. This may support that antireflux OMT acts by obtaining an anatomical change in the region of the Lower Esophageal Sphincter (LES). The patients included in this study had a long duration of GERD and desired anatomical change may be difficult to obtain by anti-reflux OMT. By using a too long interval between each treatment, LES will return to the previous situation before the next OMT and the effect will disappear.

All the five GERD-symptoms was significantly reduced one week, three and 12 months after the last OMT. The reduction in "Heartburn" and "Acid in the mouth" was most pronounced from one week after the last treatment to three and 12 months follow-up. "Epigastric pain" and "Chest pain" were nearly unchanged but "Thoracic pain" was slightly reduced. As stated above, the patients included in this study had suffered from GERD over several years. Even if the treatment, in general, prevents the reflux of acid gastric juice, the healing of the esophageal damage takes time.

PPI and H2-antagonists act by increasing the $\mathrm{pH}$ of the gastric juice, thus alleviating the damage of the oesophageal mucosa in GERD. However, the underlying cause of reflux will not be corrected, and most patients will need continuous medication for control of GERDsymptoms. The present study detected a significant reduction in the use of PPI one week, three and 12 months after the last OMT. Among the patients previously using PPI, $67.5 \%$ did not use such medication one year after the study.

GERD is a common multifactor disorder due to a defective sphincter mechanism at the esophagus-gastric junction [1]. In addition to other gastrointestinal disorder, lifestyle-related diseases, musculoskeletal disorders, asthma, fatty liver disease, and chronic diseases are among those reported to be at risk of GERD or GERD symptoms [2,3,20-22]. The mechanism of action of the anti-reflux OMT is mainly directly related to LES. It might additionally be related to musculoskeletal disorders, but more doubtfully to other diseases giving GERD symptoms.

The only approved potentially curative GERD-treatment today is surgical procedures such as fundoplication. It is a very interesting finding of the present study that OMT might have a curative effect at least on a subpopulation of GERD patients. However, some caution should be taken due to the small number of patients.

\section{Conclusion}

Anti-reflux OMT significantly decreases the GERD-symptoms and the use of PPI. 46.7 percent of the patents had no GERD symptoms one year after treatment. The estimated MED of OMT was three treatments with two days ( 48 hours) interval.

\section{Acknowledgment}

The authors would like to thank Nordic Osteopathic Research Institute, MEDOC and Norwegian Association of Osteopathy (Norsk Osteopatforbund) for their participation in the study. ClinicalTrials.gov identifier: NCT03568825

Norwegian Regional Ethical Committee number: 2016-936 


\section{References}

Cheskin LJ, Lacy BE. Healing Heartburn. John Hopkins, University Press Baltimore London, $1^{\text {st }}$ ed., p: $50-52$ (2002).

Goodall RJ, Earis JE, Coper DN, Bernstein A, Temple JG. Relationship between asthma and gastro-esophageal reflux. J. Thorax. 36(2), 116-121 (1981).

Yuksel F, Dogan M, Karatas D, et al. Clinical presentation of gastroesophageal reflux disease in children with chronic otitis media with effusion. J. Craniofacial Surg. Mar. 24(2), 380-383 (2013).

Irvine EJ. Quality of life assessment in Gastroesophageal reflux disease. Gut. 53, 35-39 (2004).

Leodolter A, Nocon M, Kulig M, et al. Gastroesophageal reflux disease is associated with absence from work: results from a prospective cohort study. World J. Gastroenterol. 11(45), 71-51 (2005).

Bjørnæs KE, Reiertsen O, Larsen S. Does osteopathic manipulative treatment have effect in treatment of patients suffering from Gastro-Oesophageal Reflux Disease? Int. J. Clin. Pharmacol. Pharmacother. 1, 116 (2016).

Chila AG, Carreiro JE. Foundations of osteopathic medicine, American Osteopathic Association (COR), England (2010).

Bjørnæs KE, Larsen S, Skauvik T, et al. The effect of Osteopathic Manipulation Therapy (OMT) in patients with
Gastroesophageal Reflux Disease (GERD). Int. J. Clin. Pharmacol. Pharmacother. 2, 132 (2013).

Larsen S, Holand T, Bjørnæs KE, et al. Randomized two-dimensional betweenpatient response surface pathway design with two interventional-and one response variable in estimating minimum efficacy dose. IJCT. 5 (2018).

Holand T, Dewi S, Larsen S. Development of a randomized twodimensional Response Surface Pathway design with two interventional- and one response variable. Open Access Journal of Clinical Trials. 8, 1-10 (2016)

Aabakken L, Larsen S, Osnes M. Visual analogue scales for endoscopic evaluation of nonsteroidal anti-inflammatory druginduced mucosal damage in the stomach and duodenum. J. Scand. Gartroenterol., 25(5); 443- 448 (1990).

Altman DG. Practical statistic for medical research. Chapman and Hall. (1991)

Agresti A. Categorical data analysis. $2^{\text {nd }}$ Ed. John Wiley and sons New Jersey. (2002)

Kleinbaum DG, Kupper LL, Nizam A et al. Applied regression analysis and other multivariate methods. Duxbury Press, $4^{\text {th }}$. Ed. Belmont Canada. (2008).

Paul RK, Rosenberger WF, Flournoy N. Quantile estimation following nonparametric phase I clinical trials with ordinal response. Stat. Med. 23(16), 2483-2495 (2004).
Stylianou M. Advanced in and limitations of up-and-down methodology: A precis of clinical use, study design and dose estimation in anesthesia research. Anesthesiology. 107(1), 144-152 (2007).

Stylianou M. Dose-finding using the biased coin up-and-down design and isotonic regression. Biometrics. 58(1) 171177 (2002).

Diniz LR, Nesi J, Curi AC et al. Qualitative evaluation of osteopathic manipulative therapy in patient with gastroesophageal reflux disease: a brief report. J. Am. Osteopath. Assoc. 114(3), 180-188 (2014).

Noll DR, Sthole HJ, Cavalier TA. Axioms, osteopathic culture, and a perspective from geriatric medicine. $J$. Am. Osteopath. Assoc. 113(12), 908-915 (2013).

Fallone CA, Mayrand S. Gastroesophageal reflux and hyperacidity in chronic renal failure. Perit. Dial. Int. 21(3), 295-299 (2001).

Catanzaro R, Calabrese F, Occhipinti $S$, et al. Nonalcoholic fatty liver disease increases the risk for gastroesophageal reflux symptoms. Dig. Dis. Sci. 59(8), 1939-1945 (2014).

Wijarnpreecha K, Panjawatanan P, Thongprayoon C, et al. Association between gastroesophageal reflux disease and non-alcoholic fatty liver disease: A meta-analysis. Saudi J.. Gastroenterol. 23(6), 311-317 (2017). 